\title{
Rotation-Invariant Pattern Recognition Approach Using Extracted Descriptive Symmetrical Patterns
}

\author{
Rehab F. Abdel-Kader ${ }^{1}$, Rabab M. Ramadan ${ }^{2}$, Fayez W. Zaki ${ }^{3}$, and Emad El-Sayed ${ }^{1}$ \\ 1 Faculty of Engineering, Port-Said University, Port-Said, Egypt \\ ${ }^{2}$ College of Computers \& Information Technology, University of Tabuk, Tabuk, KSA \\ ${ }^{3}$ Faculty of Engineering, Mansoura Universit, Mansoura, Egypt
}

\begin{abstract}
In this paper a novel rotation-invariant neural-based pattern recognition system is proposed. The system incorporates a new image preprocessing technique to extract rotationinvariant descriptive patterns from the shapes. The proposed system applies a three phase algorithm on the shape image to extract the rotation-invariant pattern. First, the orientation angle of the shape is calculated using a newly developed shape orientation technique. The technique is effective, computationally inexpensive and can be applied to shapes with several nonequally separated axes of symmetry. A simple method to calculate the average angle of the shape's axes of symmetry is defined. In this technique, only the first moment of inertia is considered to reduce the computational cost. In the second phase, the image is rotated using a simple rotation technique to adapt its orientation angle to any specific reference angle. Finally in the third phase, the image preprocessor creates a symmetrical pattern about the axis with the calculated orientation angle and the perpendicular axis on it. Performing this operation in both the neural network training and application phases, ensures that the test rotated patterns will enter the network in the same position as in the training. Three different approaches were used to create the symmetrical patterns from the shapes. Experimental results indicate that the proposed approach is very effective and provide a recognition rate up to $99.5 \%$.
\end{abstract}

Keywords- Rotation-Invariant; Pattern Recognition; Edge Detection; Shape Orientation; Image processing.

\section{INTRODUCTION}

Pattern recognition is one of the most intriguing and active research topics in the field of image processing. Its importance is due to its pervasive presence and influence in a large number of computer vision applications. In spite of the easiness of the recognition of different patterns by the human eyes, it remains challenging to implement automated pattern recognition technique that can be efficiently applied to various shapes. Research effort in this field tend to build robust techniques of pattern recognition which are insensitive to different kinds of transformations, including rotation, translation, and scale. A wide variety of techniques have been proposed to deal with specific or general instances of this problem [1]. Some techniques are based on integral transforms as invariant feature extractors. These transforms are based largely on Fourier analysis, and are probably the most common tools used in invariant pattern recognition [2-7]. Other techniques use moment functions as invariant features by taking quotients and powers of moments such as regular moments, Zernike moments, the generalized moments and Flusser and Suk's moment for affine transformation invariance [8-13]. Another group of techniques incorporates different designs of neural networks in the invariance problem which rely on the presence of symmetries among the network connections. This includes weight sharing neural network, first-order networks and higher-order neural networks [14-16]. Additional techniques that do not fit easily into any of the above categories include image normalization as in the preprocessing normalization system of Yuceer and Oflazer [17] and the symmetric filter systems [18-20].

In this paper, the proposed system is based on an image preprocessing technique which uses a newly developed efficient shape orientation method to normalize the image rotation by rotating it to a specific rotation angle. Then, the proposed image preprocessor generates a rotation-invariant descriptive pattern from the shape to be used in the training and application phases of the neural network.

\section{SHAPE ORIENTATION}

Shape orientation has emerged as an important task widely used in the area of image processing. It is very useful in a number of computer vision applications such as robot manipulation and image recognition and image registration. It is usually used as an initial step in many image processing operations. Due to the importance of the shape orientation, several techniques [21-28] are proposed to solve this problem based on different concepts like geometric moments, complex moments and principal component analysis. Some of them are area based which takes into account all points that belong to the shape. Others use only the boundary points. Although it is usually easy to determine shape orientation by the human eyes as shown in Fig. 1, it remains challenging to implement a technique which can be efficiently applied to all shapes because of the variety of shapes and their applications. Each concept used has its strengths and weaknesses relative to the processed image and the application domain. Some techniques have problems in processing symmetric shapes that have several axes of symmetry. For example the most standard technique among the area based techniques determines the shape orientation by finding the axis of the least second moment of the shape. The output axis which minimizes the integral of the squared distances from the shape points [29-31] cannot find the shape orientation of $M$-fold $(M>2)$ rotationally symmetric shapes. The same problem appears in the boundary 
based shape orientation techniques based on either the convex hull points only of the considered shape [32, 33], or the complete boundary points like [34].

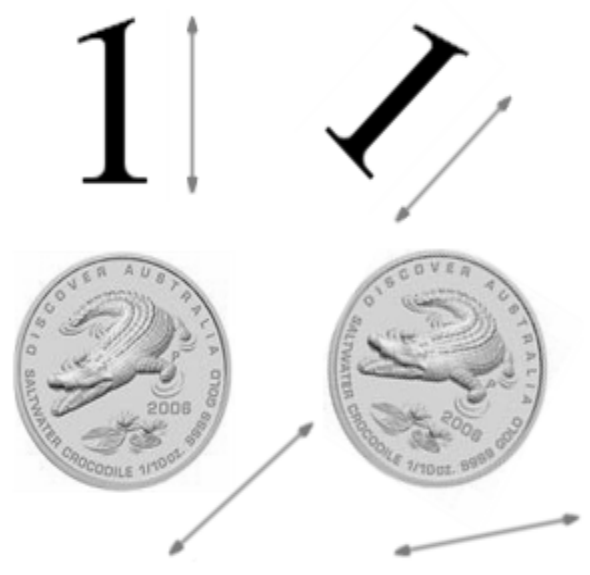

Figure 1. The shape orientation by human eye

To overcome this problem some techniques use complex or higher order moments were reported [27, 28], however they are usually computationally expensive. Alternative techniques tend to find the orientation of shapes that have equally separated multiple axes depending on the fact that in digitized images those shapes are not perfectly symmetric. This may not be practically useful due to the fact that after rotating the image, the error of the digitization rounding process may change the traced orientation of the original image. For these reasons, in the technique reported here it is concentrated on practical orientable shapes that have a unique determinable orientation. The proposed technique aims to reduce the computational cost by avoiding the use of higher order moments in cases of multiple principal axes shapes.

It is necessary for multiple principal axes shapes, to be orientable, to have non-equal angles' differences between their axes. This feature is used to define a simple method to get average of the axes angles.

\section{THE SHAPE ORIENTATION ALGORITHM}

The algorithm comprises four main steps:

1) Apply Canny edge detection [35] to the gray-level image, and obtain a binary edge map.

2) Find the center point of the pattern object $\left(x_{c}, y_{c}\right)$ by calculating the average of the vertical and horizontal coordinates of the points of the edge map.

3) Find axes of the shape which are the minimum peaks of the absolute summation of perpendicular distances from edge map points to the line passes through center :

a) The equation of the straight line that passes through the claculated center point $\left(x_{c}, y_{c}\right)$ with angle $\theta$ is given as:

$$
a x+b y+c=0 \text {. }
$$

Where

$$
\begin{cases}a=\tan \theta & \\ b=-1 & \\ c=y_{c}-x_{c} * \tan \theta & \text { if } \theta !=90 \\ a=1 & \text { if } \theta=90 \\ b=0 & \end{cases}
$$

b) The perpendicular distance $d_{i}$ from any point on the edge map $\left(x_{i}, y_{i}\right)$ to the predefined straight line is expressed as

$$
\mathrm{d}_{\mathrm{i}}=\frac{\left|a x_{i}+b y_{i}+c\right|}{\sqrt{a^{2}+b^{2}}}
$$

c) So for each angle $\theta_{j}$ in the range $\left(0 \leq \theta_{j}<\pi\right)$ stepped by any suitable step the summation of the perpendicular distances from the edge map points to the predefined line is given as

$$
\mathrm{D}_{\mathrm{j}}=\sum_{i=1}^{n} \frac{\left|a_{j} x_{i}+b_{j} y_{i}+c_{j}\right|}{\sqrt{a_{j}^{2}+b_{j}^{2}}}
$$

Where $n$ is number of edge points and the points are indexed by $i=1, \ldots n$ and $k$ is the number of the stepped angles straight lines and the lines are indexed by $j=1, \ldots k$.

d) Using resulted curve one can find the shape's axes and their angles. Where the axis is the line with angle $\theta_{j}$ which has one of the minimum peaks of summation $D_{j}$ from (4).

4) If the shape has more than one axes the averaging method could be used to find the shape orientation.

The drawn lines in Fig. 2 show the detected axes by applying the proposed technique on " 3 " as pattern in different positions as an example of single axis shapes.

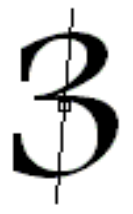

(a)

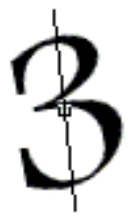

(b)

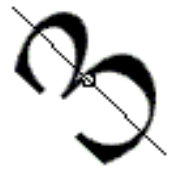

(d)

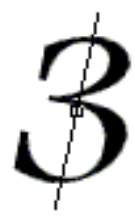

(c)

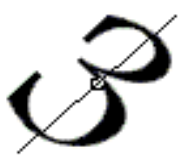

(e)
Figure 2. Samples of dominant direction angle detection

(a) Original pattern without rotation (b) After $10^{\circ}$ rotation (c) After $-10^{\circ}$ rotation (d) After $45^{\circ}$ rotation (e) After $-45^{\circ}$ rotation 
Fig. 3 shows the summation of the perpendicular distances from the edge map $D_{j}$ (on the vertical axis) to the line passes through center point with angle $\theta_{\mathrm{j}}$ (on the horizontal axis).

As shown in Fig. 3 the orientation angle is the corresponding angle of the single minimum peak value of the curve. It is clear that the phase shift between the original image curve and the rotated image curve is equal to the rotation angle. In Fig. 3(a) the original detected angle is $86^{\circ}$ and the detected angles after $\pm 10^{\circ}$ rotation are 76 and 96 with phase shift $\pm 10^{\circ}$ and also the same in Fig. 3(b). But the rotation angle and the phase shift are $\pm 45^{\circ}$.

The following points should be taken into account:

- The orientation angle from any image is equal to the output angle after $180^{\circ}$ rotation because there is no difference between the orientation directions in both cases as shown in Fig. 4(a). It is clear in Fig. 5(a) that the pattern before and after $180^{\circ}$ rotation has the same overlapped curves which results the same minimum values and output angle.

The same concept is clear in Fig. 4(b) which shows the two positions of the pattern after $\pm 90^{\circ}$ rotation and in their overlapped curves in Fig. 5(b) because the angle difference between both of them is $180^{\circ}$.

From the above section it is clear that the output angle will repeat itself every $180^{\circ}$.

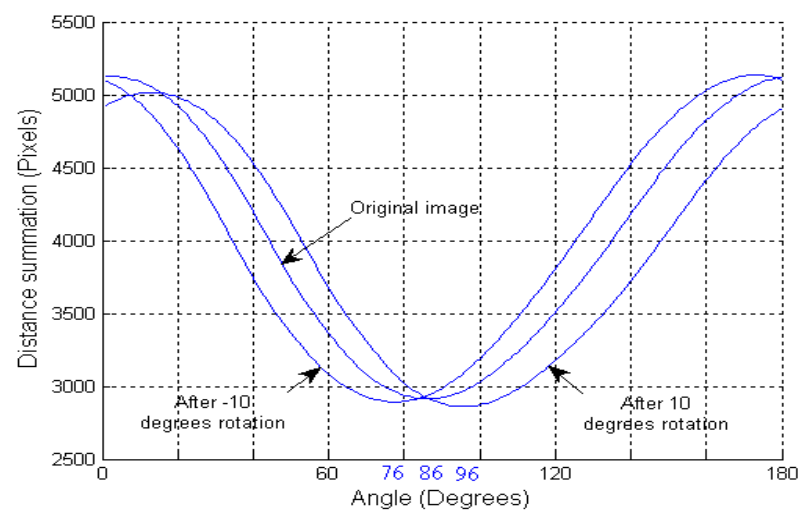

(a)

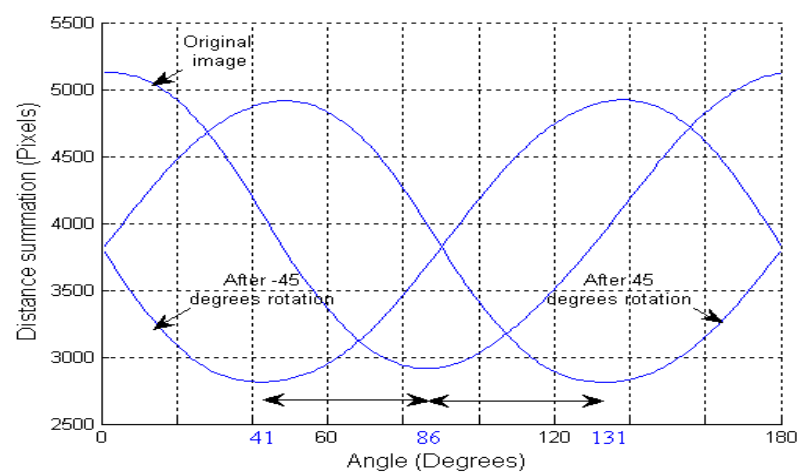

(b)

Figure 3. Relation between distance summation $D_{j}$ and the angles $\theta_{j}(a)$ Curve of $\pm 10^{\circ}$ rotated pattern (b) Curve of $\pm 45^{\circ}$ rotated pattern
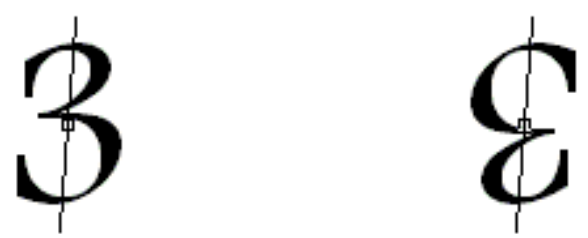

(a)
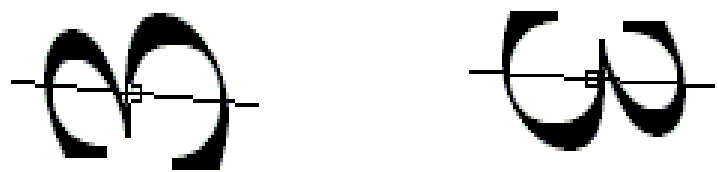

(b)

Figure 4. The repeated dominant direction every $180^{\circ}$ (a) The pattern before and after $180^{\circ}$ rotation (b) The pattern after $\pm 90^{\circ}$ rotation

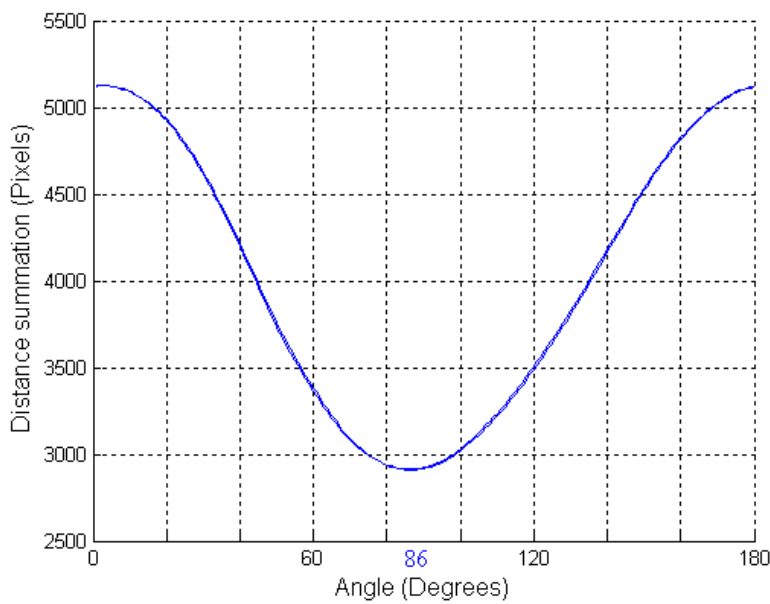

(a)

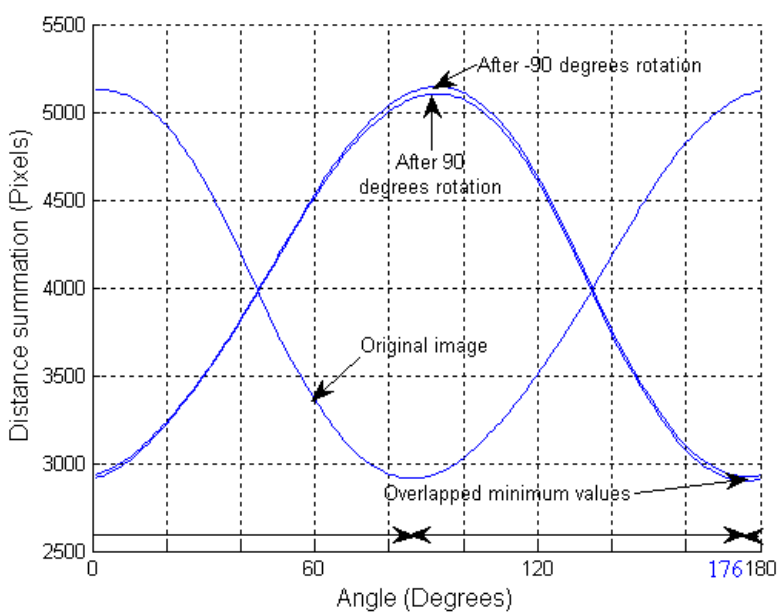

(b)

Figure 5. The overlapped curves of the pattern due to $180^{\circ}$ angle difference (a) Before and after $180^{\circ}$ rotation (b) After $\pm 90^{\circ}$ rotation 
- In some cases, the curve may have some fake minimum peaks as a result of any successive decreases and increases in value by error.

To overcome this problem it is required to get only the true minimum peaks as follows:

a) Calculate the numerical differential of the curve in order to find the global minimum candidates which are correspond to the points on the differential curve that switch from negative to positive.

b) To avoid fake candidates we find the depth of each candidate peak as the number of pixels from the candidate peak point to the nearest maximum peak. Then the depths of the found peaks are compared neglecting the candidates with depth less than a factor of the average depth.

\section{THE AVERAGING Method}

In all previous curves of " 3 " pattern there is only one minimum peak so it is sufficient to depend on finding the global minimum value of the curve because " 3 " has only one axis, in some other cases the shape may have multiple axes, so the pattern curve may have two or more minimum peaks as shown in Fig. 6(b) which shows the curve of " $X$ " pattern. This happens because the pattern has two symmetric directions to the center point.

In similar cases, previously reported techniques use higher orders moments which are computationally expensive. In the proposed approach a simple method is adopted to find the average of the axes' angles. The difficulty in using the normal average method is that each axis angle has two probable values $\theta$ and $\theta+180$. This is clear from the overlapped curves in Fig. 5. This may cause an error in the calculated average of the rotated shape due to the fact that our detected angles are always between $0^{\circ}$ and $180^{\circ}$ which means that the detected rotated angle returns to $0^{\circ}$ after passing $180^{\circ}$. This may cause a wrong average value if one or more of the angles change to the other direction after rotation, while the other angles do not.

For example the " $\mathrm{X}$ " pattern in Fig. 6 has two candidate angles $66^{\circ}$ and $114^{\circ}$ whose average is $90^{\circ}$ so after $100^{\circ}$ rotation it is expected that the average will be $190^{\circ}$ or $10^{\circ}$ (e.g. in the range given above) but the pattern will be as shown in Fig. 7, and the new two candidate angles given as $166^{\circ}$ and $34^{\circ}$ whose average is $100^{\circ}$ which is not as expected in the above argument. This happens because our expected average needs to use the angle $214^{\circ}$ instead of $34^{\circ}$ but our technique always detects the angles between $0^{\circ}$ and $180^{\circ}$.

For any number $\mathrm{m}$ of angles there are $\mathrm{m}$ different ranges that contain all angles. Each range starts with one of the angles and ends with the former one. It is clear that for the nonequally separated angles there is always a range that is narrower than the others. In our method this feature is utilized to solve the drawback of the normal averaging method. Because the differences between the angles remain the same after rotation, the narrowest range will remain the same. So to find the average we find the narrowest range that contains all angles and calculate the average of this range.
In our previous example before rotation the detected angles are $66^{\circ}$ and $114^{\circ}$ with two ranges 48 and 132 , so by using the narrowest range the average will be $90^{\circ}$. After rotation the detected angles will be $34^{\circ}$ and $166^{\circ}$ with two ranges 132 and 48 , so the angles of the narrowest range will be $166^{\circ}$ and $214^{\circ}$ with average $190^{\circ}$ or $10^{\circ}$ as shown in Fig. 7 .

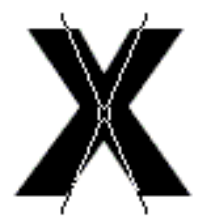

(a)

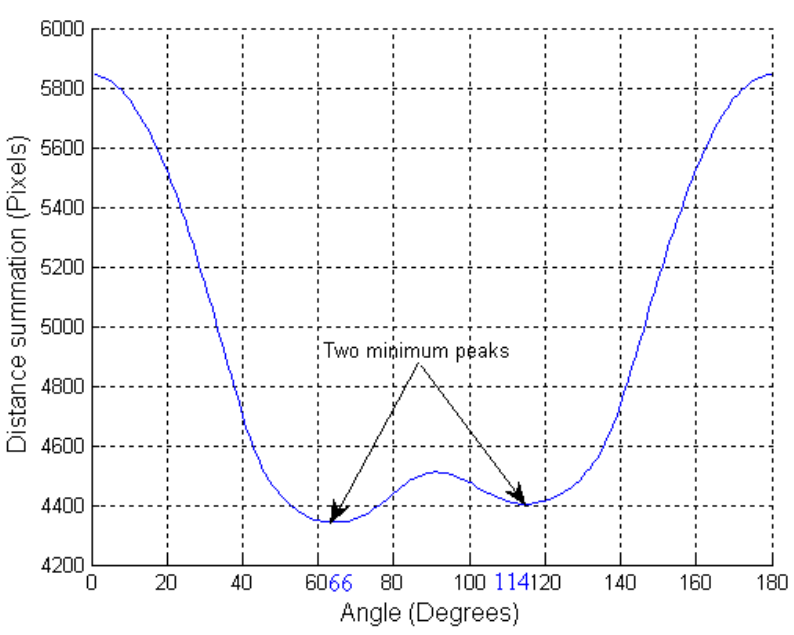

(b)

Figure 6. "X" as a sample of multiple symmetric directions (a) The two symmetric directions (b) The curve with two minimum peaks

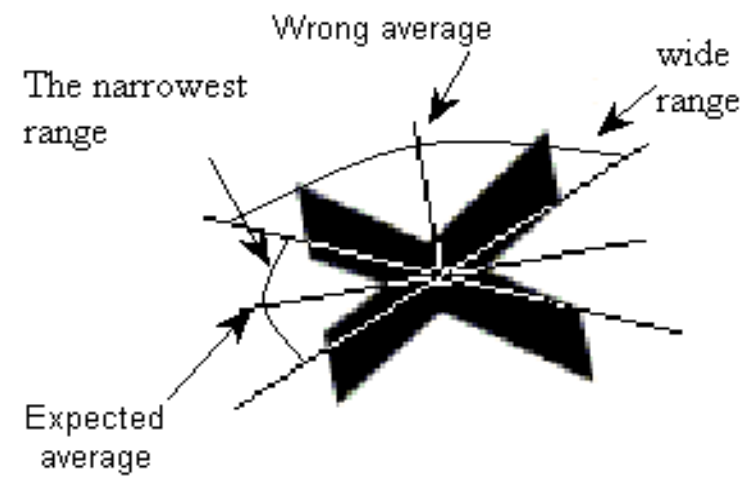

Figure 7. The average calculation method

\section{Shape OrIENTATION SiMUlation RESUlTS}

To test the shape orientation technique introduced here, more than 150 different patterns such as characters, numbers, coins and different shapes with single or multiple axes were used. The patterns in the database were rotated in all positions stepped by 5 . Moreover, the patterns have different sizes and 
different formats and use 256 gray levels for some patterns and binary images for the others. Fig. 8 shows some of the resulted orientation shapes from the proposed technique. From these results, it can be seen that the proposed technique provide accurate results at low computational cost.
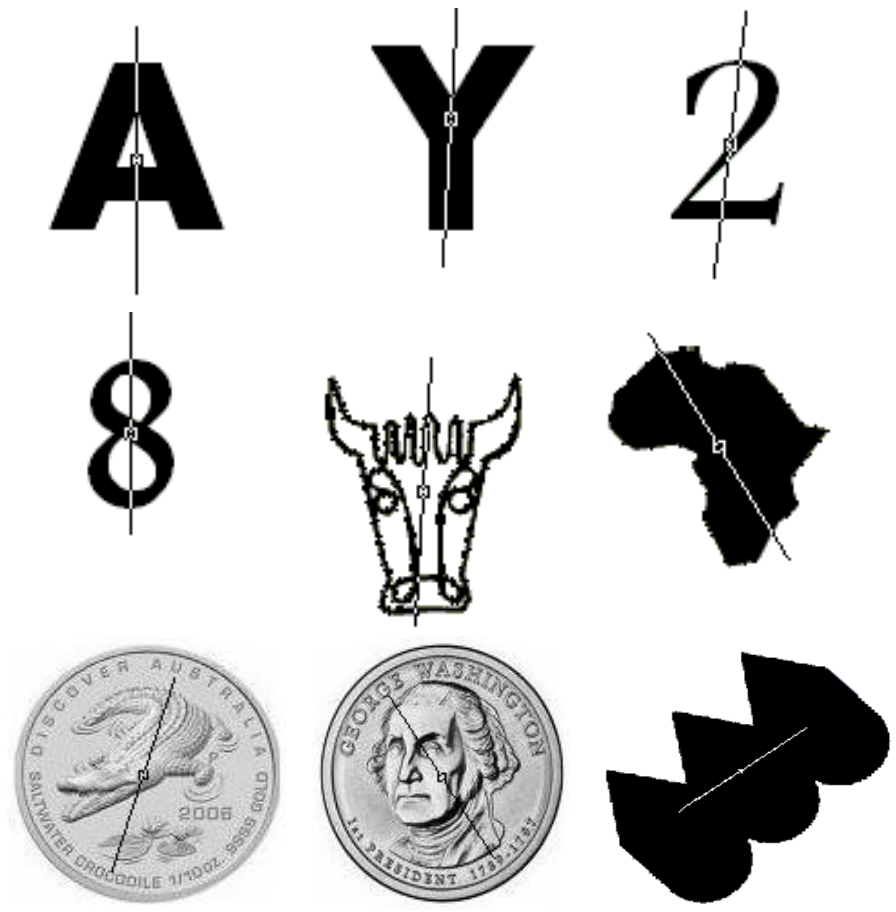

Figure 8. The detected orientations of different shapes

\section{PATTERN EXTRACTION}

The main goal of the extraction process is to create a rotation-invariant pattern from the shape which could be used in neural network training and testing to eliminate any shape rotation effect. The extracted pattern from the shape should be descriptive as much as possible, so that different shapes should have different extracted patterns to allow one to use those patterns to differentiate between the shapes.

The pattern extraction process begins by applying the shape orientation technique introduced here on the image to calculate the orientation angle of the shape. Then, the system creates a symmetrical pattern about the axis with the calculated orientation angle and the perpendicular axis on it.

The axis of symmetry of any shape should have two identical opposite sides. As the created pattern has two orthogonal axes of symmetry, it should have four identical opposite sides about the center of the shape. And so for any point in the shape to be considered as a symmetrical point, there should exist two another points lying at the same perpendicular distance from this point to the orthogonal axes but on the other sides of the axes.

We used three different approaches to create the symmetrical patterns from the shapes. The first approach is to keep only the symmetrical points about both of the orthogonal axes and eliminate the other points. By using this approach, the extracted pattern is the result of subtracting the nonsymmetrical points from the shape. Fig. 9(c) shows some samples of the extracted patterns using this approach.

Unlike the first approach, the second approach is to complement the nonsymmetrical points by adding their corresponding reflected points about the axes to the shape. In this way the nonsymmetrical points are converted into symmetrical points instead of eliminating them. Fig. 9(d) shows some samples of the extracted patterns using the second approach.

Although, the extracted patterns in the previous two approaches were based on the input shapes; there is still possibility to find two different shapes having the same extracted pattern. In order to reduce this possibility and to make the extracted pattern more descriptive, a hybrid method (third approach) using the extracted descriptive information from the previous two approaches. That is, the extracted pattern by the third approach is the result of subtracting the first method symmetrical pattern from the second method complemented pattern. In this way the possibility of finding two different shapes having the same extracted pattern is reduced because it is difficult for the different shapes to have the same subtracted and complemented patterns in the same time. It is clear from Fig. 9(e) that the extracted pattern using the third approach is the result of subtracting the pattern in Fig. 9(c) from the pattern in Fig. 9(d).

Due to the symmetry of the previous extracted patterns about their axes, the extracted patterns of any two opposite positions of any shape are the same. The importance of this feature is that we can't cancel the effect of shape rotation using only the shape orientation to rotate the shape to any reference angle because the orientation angle of any shape will be the same after $180^{\circ}$ shape rotation because there is no difference between the orientation directions in both cases as shown in Fig. 4. Therefore, one can't ensure that the resulted rotated shape would have the required reference angle as it could be in its opposite direction. But extracting a symmetrical pattern after rotating the shape to a reference angle will have the same pattern in both cases as shown Fig. 10 which could be used in both of the training and test phases of the neural network. In this way one may ensure that the same pattern would be applied to the network in both phases whatever the rotation angle of the shape is. 

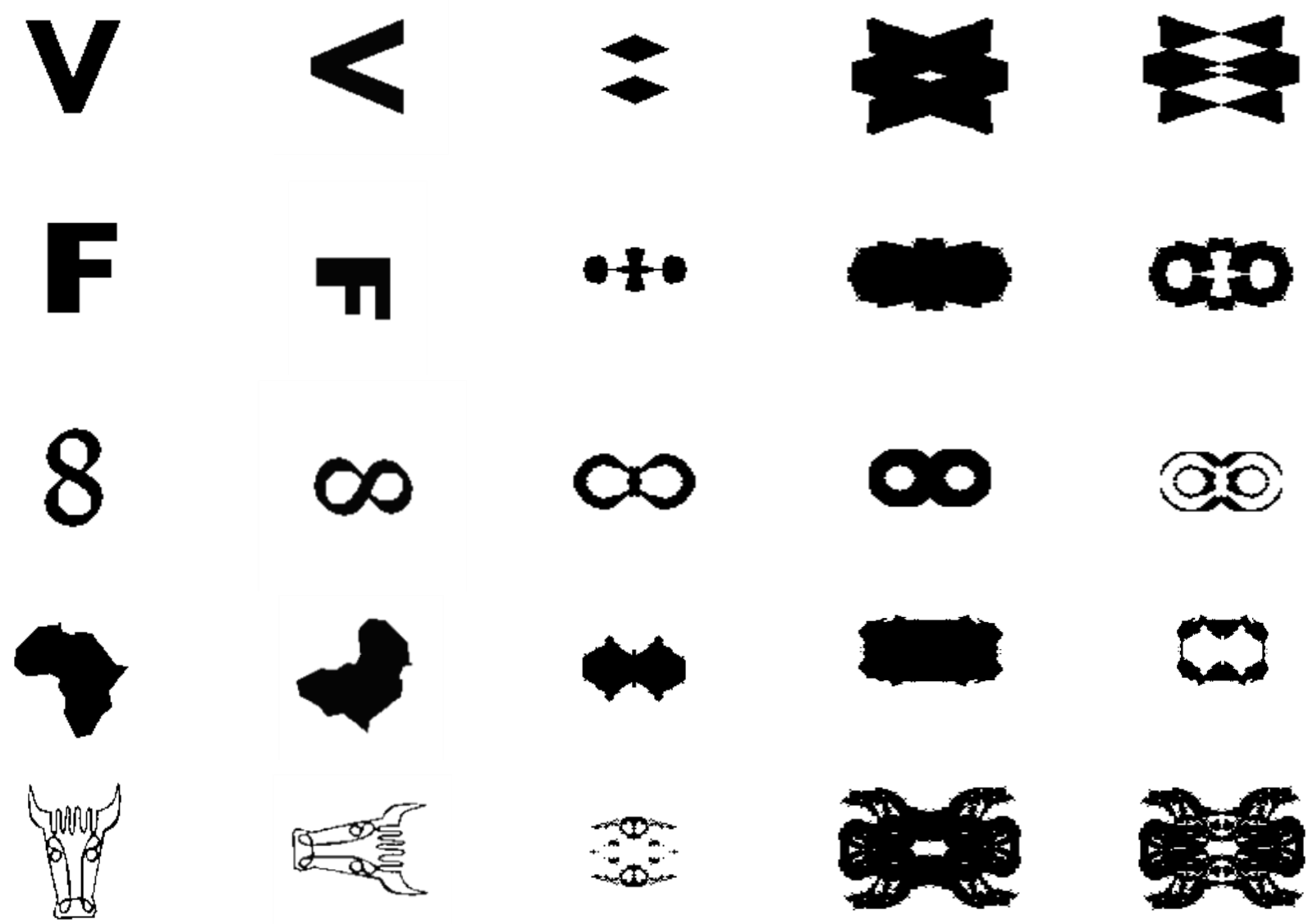

(a)

(b)

(c)

(d)

(e)

Figure 9. some samples of the extracted patterns using the three different approaches (a) The input shape (b) The shape after being rotated to have 0 orientation angle (c) The extracted pattern using the first approach (d) The extracted pattern using the second approach (e) The extracted pattern using the third approach
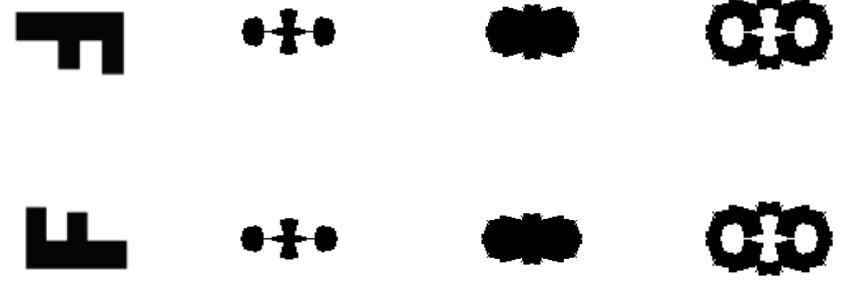

Figure 10. The three extracted patterns of the shape of ' $\mathrm{F}$ ' in the $0^{\circ}$ orientation angle position and in $180^{\circ}$ orientation angle position

\section{ROTAION INVARIANT NEURAL PATTERN RECOGNITION SYSTEM}

Fig. 11 shows the different stages of the rotation invariant neural-based pattern recognition system. First, the proposed shape orientation technique is applied on the training image to get its orientation angle $\theta$. Second, the image is rotated to adapt its orientation angle to any fixed reference angle. In our implementation, the system rotates the image by $(180-\theta)^{\circ}$ to ensure that the new orientation angle will be $0^{\circ}$ or $180^{\circ}$. Third, the system extracts the symmetrical pattern of the shape using one of the three proposed approaches.

Fourth, a feature extraction technique is applied on the pattern to get the input vector of the shape to be applied to the back propagation neural network. In the simulation, the images are down-sampled to a suitable size and then PCA feature selection technique is applied to reduce input vector dimensions while preserving most of the image details.

\section{THE SYSTEM EXPERIMENTAL RESULTS}

The performance of the system is evaluated using 60 different shapes. An image database containing rotated shapes with different rotation angles from $0^{\circ}$ to $355^{\circ}$ stepped by $5^{\circ}$ was utilized in the experiments. Two different testing models were applied on the system, in the first model only the pattern extracted from the original position of the shape without rotation was used in the training phase.

Table 1 presents the average recognition rates of the system using the different proposed symmetrical patterns with only one pattern per shape used in the training. 


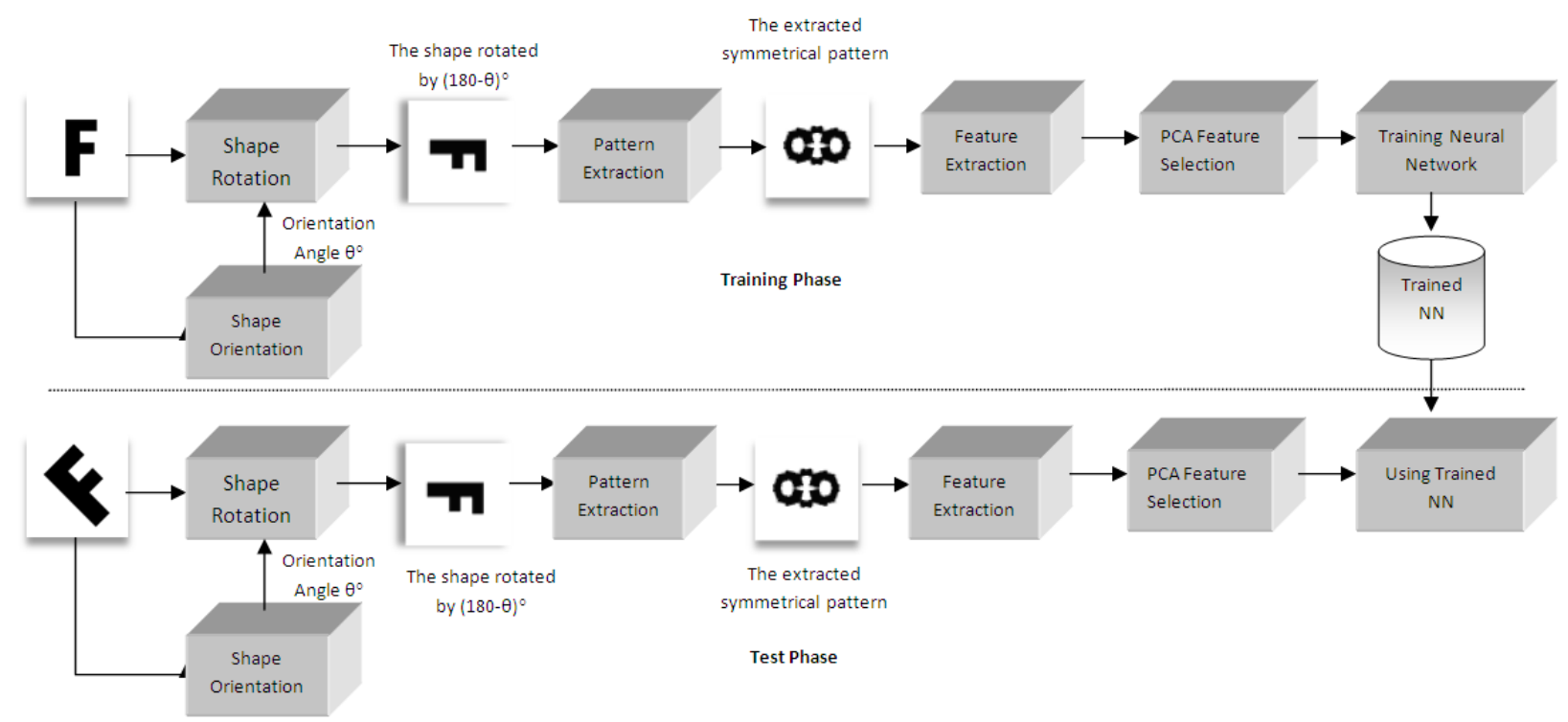

Figure 11. Block diagram of the rotation invariant neural-based pattern recognition system.

TABLE I. PERFORMANCE OF THE PROPSED SYSTEM USING DIFFERENT PATTERN EXTRACTION APPROACHES WITH THE FIRST TESTING MODEL

\begin{tabular}{|c|c|}
\hline Pattern Extraction Approach & Recognition Rate \\
\hline $\begin{array}{c}\text { First approach (using only } \\
\text { symmetrical points of the shape) }\end{array}$ & $91 \%$ \\
\hline $\begin{array}{c}\text { Second approach (complementing } \\
\text { nonsymmetrical points of the shape) }\end{array}$ & $89 \%$ \\
\hline Third approach (the hybrid method) & $94 \%$ \\
\hline
\end{tabular}

In the second model the neural network was trained using four patterns for every shape extracted at different rotation angles. Table 2 shows the results of the second testing model with high improvement in the performance.

\section{TABLE II. PERFORMANCE OF THE PROPSED SYSTEM USING DIFFERENT PATTERN EXTRACTION APPROACHES WITH THE SECOND TESTING MODEL}

\begin{tabular}{|c|c|}
\hline Pattern Extraction Approach & Recognition Rate \\
\hline $\begin{array}{c}\text { First approach (using only } \\
\text { symmetrical points of the shape) }\end{array}$ & $95 \%$ \\
\hline $\begin{array}{c}\text { Second approach (complementing } \\
\text { nonsymmetrical points of the shape) }\end{array}$ & $92 \%$ \\
\hline Third approach (the hybrid method) & $99.5 \%$ \\
\hline
\end{tabular}

As expected, the third approach has the best recognition rate because it uses the extracted information of both of the other two approaches which makes its extracted patterns much more descriptive and reduces the possibility of having the same pattern from different shapes.

\section{CONCLUSIONS}

In this paper, a rotation-invariant neural pattern recognition system based on an image preprocessing technique to extract a rotation-invariant descriptive pattern from the shapes is presented. The system utilizes a newly proposed algorithm for finding the orientation of shapes that have several non-equally separated axes of symmetry. An effective averaging method is presented to calculate the average angle of the shape's axes of symmetry without any need to use higher orders techniques to reduce the computational cost. The orientation angle is used to normalize the shape image and eliminate any rotation effect before the feature extraction phase. Furthermore, three different approaches to extract the symmetrical patterns are proposed.

\section{REFERENCES}

[1] Wood, J., Invariant pattern recognition: A review, Pattern Recognition 29 (1996) 1-17.

[2] Rehab F. Abdel-Kader, Rabab M. Ramadan, Rawya Y. Rizk, Rotation Invariant Face Recognition Based on Hybrid LPT/DCT Features, International Journal of Electrical and Computer Engineering 7 (2008) 488.

[3]. V. Fayn, V. Sorokin and V. Vaynshteyn, Continuousgroup, pattern recognition, Engng Cybern. 6(1969) 97-106.

[4] T. M. Caelli and Zhi-Qiang Liu, On the minimum number of templates required for shift, rotation and size invariant pattern recognition, Pattern Recognition 21(1988) 205-216.

[5] R. N. Bracewell, The Fourier Transform and Its Applications, Electrical and Electronic Engineering Series, McGraw-Hill Book Company (1978).

[6] E. Brigham, The Fast Fourier Transform and Its Applications. PrenticeHall (1988).

[7] M. Clausen and U. Baum, Fast Fourier Transforms, Wissenschaftsverlag, Mannheim (1993).

[8] Y. Li, Reforming the theory of invariant moments for pattern recognition, Pattern Recognition, 25(1992) 723-730.

[9] S. Perantonis and P. Lisboa, Translation, rotation and scale invariant pattern recognition by high-order neural networks and moment classifiers, IEEE Trans. Neural Networks, 3(1992) 241-251.

[10] A. Khotanzad and J.-H. Lu, Classification of invariant image representations using a neural network, IEEE Trans. Acoustics, Speech and Signal Process, 38(1990) 1028- 1038.

[11] A. Khotanzad and Y. H. Hong, Invariant image recognition by Zernike moments, IEEE Trans. Pattern Analysis Mach. Intell, 12(1990) 489-49. 
[12] A. Shvedov, A. Schmidt and V. Yakubovich, Invariant systems of features in pattern recognition, Automation Remote Control, 40(1979) 131- 142.

[13] J. Flusser and T. Suk, Pattern recognition by affine moment invariants, Pattern Recognition, 26(1993) 167-174.

[14] L. Fausett, Fundamentals of Neural Networks, Prentice-Hall, Englewood Cliffs, 1994.

[15] J. Hertz, A. Krogh and R. Palmer, Introduction to the Theory of Neural Computation, Addison-Wesley (1991).

[16] M. Minsky and S. Papert, Perceptrons. MIT Press, Cambridge, (1969).

[17] C. Yuccer and K. Oflazer, A rotation, scaling and translation invariant pattern classification system, Pattern Recognition, 26(1993) 687-710.

[18] R. Lcnz, Group invariant pattern recognition, Pattern Recognition 23(1990) 199-217.

[19] R. Lenz, Group Theoretical Methods in Image Processing, volume 413 of Lecture Notes in Computer Science, Springer-Verlag (1990).

[20] J. Mas and E. Ramos, Symmetry processing in neural network models, J. Physics A 22(1989) 3379-3391.

[21] J. Cortadellas, J. Amat, F. de la Torre, Robust normalization of silhouettes for recognition application, Pattern Recognition Lett. 25 (2004) 591-601.

[22] V.H.S. Ha, J.M.F. Moura, Afine-permutation invariance of 2-D shape, IEEE Trans. Image Process. 14 (11) (2005) 1687-1700.

[23] J.-C. Lin, Universal principal axes: an easy-to-construct tool useful in defining shape orientations for almost every kind of shape, Pattern Recognition 26 (1993) 485-493.
[24] J.-C. Lin, The Family of Universal Axes, Pattern Recognition 29 (1996) 477-485.

[25] D. Shen, H.H.S. Ip, Generalized affine invariant normalization, IEEE Trans. Pattern Anal. Mach. Intell. 19 (5) (1997) 431-440.

[26] D. Shen, H.H.S. Ip, K.K.T. Cheung, E.K. Teoh, Symmetry detection by generalized complex (GC) moments: a close-form solution, IEEE Trans. Pattern Anal. Mach. Intell. 21 (5) (1999) 466-476.

[27] W.H. Tsai, S.L. Chou, Detection of generalized principal axes in rotationally symmetric shapes, Pattern Recognition 24 (1991) 95-104.

[28] J. Žuni'c, L. Kopanja, J.E. Fieldsend, Notes on shape orientation where the standard method does not work, Pattern Recognition 39 (5) (2006) 856-865.

[29] B.K.P. Horn, Robot Vision, MIT Press, Cambridge, MA, 1986.

[30] R. Jain, R. Kasturi, B.G. Schunck, Machine Vision, McGraw-Hill, New York, 1995.

[31] R. Klette, A. Rosenfeld, Digital Geometry, Morgan Kaufmann, San Francisco, 2004.

[32] H. Freeman, R. Shapira, Determining the minimum-area encasing rectangle for an arbitrary closed curve, Comm. of the ACM 18 (1975) 409-413.

[33] R.R. Martin, P.C. Stephenson, Putting objects into boxes, Computer Aided Design 20 (1988) 506-514.

[34] J. Žuni'c, M. Stojmenovic, Notes on shape orientation where the standard method does not work, Pattern Recognition 41 (5) (2008) 1785-1798.

[35] J. Canny, A Computational Approach To Edge Detection, IEEE Trans. Pattern Analysis and Machine Intelligence, 8 (6)(1986) 679-698. 